\title{
Application of Humanistic Nursing and Endocrine Nursing
}

\author{
Yuhong $\mathrm{YaO}^{1}$, Xia Wu ${ }^{2}$, Yaohui Deng ${ }^{1}$, Qianqian Yao ${ }^{3}$ \\ ${ }^{1}$ Henan Province Hospital of TCM, Zhengzhou, Henan, 450002 \\ ${ }^{2}$ Henan Province Coal General Hospital, Zhengzhou, Henan, 450002 \\ ${ }^{3}$ The first affiliated hospital of Henan university of TCM, Zhengzhou, Henan, 450002
}

Keywords: Humanistic Nursing, Endocrine Nursing, Application

\begin{abstract}
With the development of the economy, social progress, people's living standards are also rising, at the same time, people's health care requirements become higher and higher, not only requires the quality and efficacy of medical care, while more attention to health care process In the service, the demand for humanistic care is also increasing. In endocrine care, humanistic care is particularly important. With the development of the times, humanistic nursing has gradually become the most important nursing form and development trend in modern nursing.
\end{abstract}

\section{Introduction}

With the new round of medical service reform, and the people's health needs gradually increased, especially for the medical treatment process care services are also very concerned about, and human care model is very fit with the needs of patients with care services. Humanistic care refers to the care of the care process to patients with care as the main body, the implementation of humanistic care, through a series of modern care reflects the care of patients, so as to build a friendly, mutual trust and harmonious nurse-patient relationship. In endocrine disease care, patients not only need good medical technology, but also on the medical care process has certain requirements.

Humanistic nursing is to create a spirit of inspiring patients, understanding patients, respect for patients, caring for patients with hospital culture, the patient as a center of the humanities environment humanistic care services to show the spirit of good care, professionalism and style of work, created A stable and comfortable hospital culture environment, effective implementation of all services for patients, in order to patients all the care service concept, to the hospital to establish a high quality social image, to achieve social benefits, economic benefits win-win goal $\$$ in the hospital for the provision of human Care services in the process, should also promote the direction of patient health as the center. Humanistic care services enable patients to obtain physical care, psychological, spiritual, life and safety and other care and care, so that patients get a greater sense of security, satisfaction, in order to help ensure the patient's mind is stable, the clinical treatment, the prognosis To a positive role in promoting.

\section{Characteristics and Significance of Humanistic Nursing}

In the endocrine care, the humanistic care is very important, and the concrete embodiment of human care is in the nursing work, nurses on the human care of the patient, its connotation is people-oriented, that is, in nursing work, nurses to patients, patients, care for patients, take care of the patient's emotions, but also to learn their own knowledge in the form of care to the patient, so that in the pain feel the beauty of human beauty. Humanistic care is evolved by Watson's "human care" nursing model, in which the process of humanistic care is explained in detail. Some people said: patients without medical and will be caught in the hope; patients without care and will be caught in helplessness. Thus, doctors and nurses are complementary, indispensable, in order to better coordinate the relationship between doctors and patients and it must be done with medical care. With the development of the economy, the cause of nursing is also booming, people also put forward higher demands on the content of nursing services, not only to take care of the patient's condition, so that it can be better recovery, but also respect for patients, understand them Feel the 
protection of their personality and privacy. For the patients to make reasonable demands, should try to meet the patient as a loved one, to give its meticulous care, the real realization of humanistic care. In today's clinical care work, to be human care, we must be in the heart of the patient as a loved one, consciously patient-centered, to create a care for patients, care for patients, the patient responsible environment, with perfect service, to patients Care, so humane service, and ultimately to achieve humanistic care. Endocrine patients have their own characteristics, need to be early detection and treatment, at the same time, a safe environment for patients is extremely important. So timely and effective treatment and care, safe and comfortable environment and targeted health guidance, has become the most important endocrine treatment of treatment. In endocrine care, the need to patient demand-oriented, to provide a most comfortable environment, so that patients and their families are satisfied, so that patients more peace of mind to receive treatment.

\section{Research Data and Methods}

A total of 120 patients were enrolled in the Department of Endocrinology from August 2014 to October 2014, including 64 males and 56 females, mean age (40.16 \pm 5.04$), 120$ patients Including: 20 patients with hyperthyroidism, 5 patients with hypothyroidism, 90 patients with diabetes, 5 patients with gout; two months during the selected 120 patients were divided into observation group and control group, the two groups The patients were divided into two groups according to the general clinical data of sex, age and disease type. There was no significant difference between the two groups $(\mathrm{P}>0.05)$, which was clinically comparable.

(1) the control group: the group of patients according to the type of disease and the degree of disease routine nursing, the main contents include: the patient's blood pressure, pulse, breathing and body temperature and other life line strict monitoring, and patients taking the relevant treatment Disease trends and drug reactions were observed, if the patient appeared a more obvious abnormal situation, the patient should be the first time the basic situation reported to the attending physician, and with its targeted treatment; for patients to create a good treatment and rehabilitation environment, On time on the ward for disinfection and cleaning, scientific control of the ward temperature, humidity and lighting and ventilation conditions.

(2) observation group: the group of patients in the conventional nursing on the basis of the introduction of humanistic care intervention, the specific content includes: from this study, the vast majority of patients for their own diseases and hospital treatment plan is not the correct understanding and understanding, So in the treatment process is prone to tension, fear and other negative psychological mood, improve the quality of care, the large number of patients, which is a basic status of endocrinology, in order to ensure that patients can receive better care services, we asked us The patient's diagnosis and treatment time for scientific arrangements in the morning rounds can be based on the patient's condition to the effective timing of the disease for the relatively serious patients, may be appropriate to increase the time and the number of personnel for the various questions raised by patients, Medical staff to conduct a detailed answer, and the process of answering should be avoided as much as possible to use too much professional words, in order to avoid the patient's understanding of the deviation.

The evaluation of the compliance of the two groups of patients during the course of treatment was evaluated. Then, the patient's health knowledge and nursing satisfaction were evaluated by questionnaire.

SPSS.17.0 statistical software to analyze the use of $(\mathrm{x}- \pm \mathrm{s})$ that the measurement of the data, and the application of paired t test, the difference was statistically significant $\mathrm{P}<0.05$.

The hospitalization time was $(10.87 \pm 4.49) d$ in the observation group $(n=60)$ and $(16.12 \pm$ 5.35) $\mathrm{d}$ in the control group $(\mathrm{n}=60)$, and the hospitalization time $(\mathrm{T}=5.8224, \mathrm{P}=0.0000)$. The difference between the two groups was statistically significant $(t=5.8224, \mathrm{P}=0.0000)$.

Comparison of treatment compliance and health knowledge among the two groups: the criteria for assessing the compliance of treatment were: the ability to take drugs in accordance with the requirements of the medical staff and the nursing staff to carry out the relevant care treatment; 
health knowledge awareness rate The criteria were as follows: Through the questionnaire survey, the patients had more than $70 \%$ of the relevant knowledge of their own diseases. By data integration, the compliance rate of the observation group was better than that of the control group. The difference was statistically significant significance.

\section{The Specific Measures for the Application of Humanistic Care in Endocrine Care}

Endocrine care in the application of humanistic care is very important. First of all, we should create a warm and patient environment for patients. The importance of the environment to the patient slowly discovered by people, a comfortable and warm environment can give patients a better treatment of psychological, to reduce the pressure, you can relax, do not bear too much mental burden, but also make it The sense of trust and dependency on the nurse, such emotions are conducive to its recovery. Ward wall color can be replaced by fresh green, green symbolizes life, is conducive to patient adjustment mood. But also in the wall decorated with some oil paintings, and even some of the paintings of patients, has recovered patients to send gifts. When a patient is admitted to hospital, they can tell them to listen, to enhance their confidence in recovery. Focus on improving and enhance their own quality, lay a good foundation for good service. Humanity care in the proportion of endocrine care is very important, but also the perfect realization of the nursing work to achieve an important guarantee. The hospital should focus on the training of nurses, the development of nurses must master the theoretical knowledge, regular nurses to learn; can also carry out lectures to enhance the quality of nurses. The hospital can organize a number of activities to strengthen the nurses' learning ability. The hospital can arrange a number of qualified nurses in the endocrine care in the tour, so that more people understand the disease, learn to maintain. In the application of humanistic care in endocrine care, the most important thing is to respect and care for patients. Even if the patient is the same disease, its needs are not the same. Hospitals should develop different plans to help them better recover according to the needs of different patients.

Nursing staff is a clinical nursing professional workers, the key task is to help patients to restore health, to help healthy people to maintain health, humanistic spirit is mainly concerned about people, respect for people to meet the basic requirements of health care, nursing staff in clinical care services, Regardless of what level of patients in patients, regardless of the severity of patients, should respect, care for patients, treat life, as far as possible to meet the needs of patients with human care services, which is a necessary factor in human care. In the endocrine department nursing work to carry out humanistic care, for different patients to do the urgency of urgency, want patients to think, try to ensure that the basic rights and interests of patients, enhance patient health care, prevention and treatment in order to meet the reasonable needs of patients, Improve the satisfaction of nursing services, so that patients with significant increase in compliance, to promote the improvement of clinical efficacy, is conducive to the friendly development of nurse-patient relationship. At the same time, but also to mobilize the enthusiasm and enthusiasm of health care workers work, showing the self-worth of health care workers, so that the subjective initiative of health care personnel to fully play out and reflects the brand service.

\section{Conclusion}

Combined with clinical practice, the quality of care will be largely related to the health and safety of patients, therefore, combined with their own actual situation, and constantly adjust and improve the nursing methods, the hospital reflects the social function of one of the important methods The research method has been tested scientifically and it is worthy of reference in clinical practice. In today's world economy, the rapid development of culture today, personal health has become the most concerned about the issue. How to do the best care of the hospital is also concerned about the issue, only to allow patients to really get the most intimate care in order to care for the role of care to the maximum. This nursing work will be done well and bad, related to the safety of patients, so that patients get safe and efficient care services, but also an important manifestation of humanistic care. 


\section{References}

[1] Peng Rongfen. Application value of humanistic care in endocrine clinical nursing [J]. Journal of Health, 2014 (01)

[2] Wang Xia, Shen Xiao. Humanistic nursing in elderly patients with diabetes [J] .Qi Lu Nursing Journal, 2014 (01)

[3] Yang Tiantan, Zhou Yan, Zhao Mengyuan. 5 kinds of core journals published humanistic care research literature analysis [J]. Journal of Nursing, 2013 (07)

[4] Jia Yanjie, Zhu Dan. Humanistic nursing in the application of [J]. Nursing Journal, 2006 (17)

[5] Wang Qinghua, Liu Huaxia. Watson human care theory [J]. Nursing Research, 2004 (23)

[6] Wu Yajun, Feng Jin'e. Humanistic nursing and nursing care behavior [J]. Practical Nursing Journal, 2003 (05) 